\title{
Flat Fading in Mittelwellen-DRM-Gleichwellennetzen
}

\author{
B. Müller und J. Philipp
}

Südwestrundfunk, Stuttgart, Germany

Zusammenfassung. Digitale Rundfunksysteme mit OFDMMehrträgerverfahren wie T-DAB, DVB-T und DRM wurden speziell entwickelt, um auch bei Mehrwegeausbreitung einen guten Empfang zu gewährleisten. Mit diesem Verfahren wurde auch der Aufbau von Gleichwellennetzen (SFNs) möglich. In solchen Gleichwellennetzen wird durch die Überlagerung der Signalanteile von unterschiedlichen Sendern mit verschiedenen Laufzeiten ein frequenzselektives Fading verursacht. Diese Überlagerung beeinträchtigt die Empfangsqualität nicht, solange die Kohärenzbandbreite (Kehrwert des Laufzeitunterschiedes) klein gegenüber der Signalbandbreite ist. Diese Bedingung ist meist bei den Systemen T-DAB und DVB-T erfüllt, bei denen die Signalbandbreite in der Größenordnung von $10^{6} \mathrm{~Hz}$ liegen. Im DRMSystem ist dagegen die Signalbandbreite auf wenige $\mathrm{kHz}$ beschränkt. Dadurch kann das ,selektive“ Fading leicht das komplette Signal beeinträchtigen und somit bei großen Wellenlängen auch weite Gebiete erfassen. Zur Klärung, welche Maßnahmen getroffen werden können, um dennoch ein robustes DRM-Gleichwellennetzes auf Mittelwelle zu betreiben, wurden die Interferenz-Effekte eines Gleichwellennetzes mit zwei Sendern theoretisch modelliert und durch Feldmessungen in einem realen Gleichwellennetz mit zwei synchron arbeitenden Sendern im Gebiet zwischen Kaiserslautern und Mainz untersucht.

Abstract. Multicarrier digital broadcasting systems like TDAB, DVB-T and DRM are designed to provide reception especially in multipath environments. At the same time this feature enables the implementation of single frequency networks (SFNs). In these, the superposition of the signals arriving from the different transmitters with different delays gives rise to interference in the form of frequency selective fading. This does not impair reception quality as long as the coherence bandwidth (the reciprocal of the time delay dif-

Correspondence to: B. Müller

(bernd.mueller@swr.de) ference) is small compared to the signal bandwidth. This condition in most cases is safely met in T-DAB and DVB$\mathrm{T}$ networks where the signal bandwidth is of the order of $10^{6} \mathrm{~Hz}$. In the DRM system the signal bandwidth however is confined to a few kHz only. Thus ,,selective“ fading may easily affect the whole signal and, due to the large wave length, extend to comparatively large areas. To clarify which provisions have to be met to nevertheless render the operation of medium wave DRM SFNs feasible the interference effects in a two-transmitter SFN have been modelled theoretically and evaluated by field measurements in a real network consisting of synchronized transmitters sited near Kaiserslautern and Mainz.

\section{Theoretischer Hintergrund}

In einem ausgewogenen Gleichwellennetz von zwei DRMSendern entsteht in der Mitte zwischen den beiden Senderstandorten ein Verwirrungsgebiet, in dem sich die Signale derart überlagern, dass erhebliche Feldstärkeeinbrüche entstehen. Diese zu erwartenden Einbrïche lassen sich für ein ideales Gleichwellennetz theoretisch berechnen. Bei der folgenden Betrachtung wird die für einen Empfänger verfügbare Gesamtleistung des interferierenden OFDM-Signals (siehe z.B. (Mäusel, 2002)) im Versorgungsgebiet untersucht und eine entsprechende Formel hergeleitet.

Bei der Herleitung geht man davon aus, dass von beiden Sendern zeitgleich (synchron) dasselbe Signal ausgesendet wird und das E-Feld vertikal polarisiert ist. Das angenommene OFDM-Signal mit der Bandbreite $B$ um die Mittenfrequenz $f_{0}$ (vgl. Abb. 1) besteht aus einer festen Anzahl von Trägern, deren Phasenlage und Amplitude während der Symboldauer konstant sein sollen (z.B. DRM mit 4 QAMModulation (ETSI, 1989), bei höherwertigen Modulationsarten kann man mit dem statistischen Mittelwert der Trägeramplituden über mehrere Symbole rechnen). 


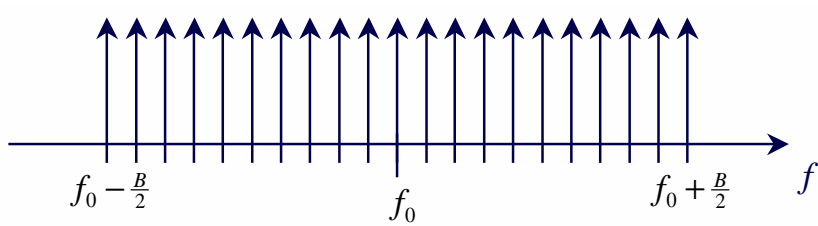

Abb. 1. Spektrum eines OFDM-Signals der Bandbreite $B$.

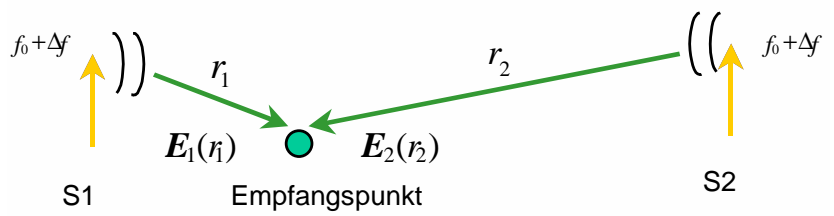

Abb. 2. Überlagerung zweier Einzelträger am Empfangspunkt.

Die elektrische Feldstärke eines Einzelträgers $\left(f_{0}+\Delta f\right)$ ergibt sich am Empfangspunkt $r$ nach der bekannten Wellengleichung (Meinke, 1992) zu:

$\boldsymbol{E}(r, t)=\hat{E}(r) e^{j 2 \pi\left(f_{0}+\Delta f\right)\left(t+\frac{r}{c_{0}}\right)}$

Für das überlagerte Summenfeld zweier Einzelträger (vgl. Abb. 2) erhält man damit:

$$
\begin{aligned}
\boldsymbol{E}_{12}\left(r_{1}, r_{2}, t\right)= & \hat{E}_{1}\left(r_{1}\right) e^{j 2 \pi\left(f_{0}+\Delta f\right)\left(t+\frac{r_{1}}{c_{0}}\right)}+ \\
& +\hat{E}_{2}\left(r_{2}\right) e^{j 2 \pi\left(f_{0}+\Delta f\right)\left(t+\frac{r_{2}}{c_{0}}\right)}
\end{aligned}
$$

Innerhalb einer Symboldauer können wir konstante Verhältnisse annehmen, dadurch ergibt sich eine mittlere Leistung des Summensignals während der Symboldauer zu:

$$
\begin{aligned}
\left|\boldsymbol{E}_{12}\right|^{2} \sim & \hat{E}_{1}\left(r_{1}\right)^{2}+\hat{E}_{2}\left(r_{2}\right)^{2}+ \\
& +2 \hat{E}_{1}\left(r_{1}\right) \hat{E}_{2}\left(r_{2}\right) \cos \left(2 \pi \frac{\left(f_{0}+\Delta f\right)}{c_{0}}\left(r_{2}-r_{1}\right)\right)
\end{aligned}
$$

Betrachtet man die Überlagerung aller Einzelträger innerhalb der Bandbreite $B$ des OFDM-Signals (gleiche Amplitude $\hat{E}$ ) und berechnet die bandbreitenbezogene verfügbare Leistung im E-Feld mit

$$
P_{12}=\int_{-\frac{B}{2}}^{+\frac{B}{2}}\left|\boldsymbol{E}_{12}\right|^{2} d \Delta f,
$$

so lässt sich folgende Gleichung herleiten:

$$
\begin{aligned}
& \frac{P_{12}}{B} \sim \hat{E}_{1}\left(r_{1}\right)^{2}+\hat{E}_{2}\left(r_{2}\right)^{2}+ \\
& +2 \hat{E}_{1}\left(r_{1}\right) \hat{E}_{2}\left(r_{2}\right) \cos \left(2 \pi \frac{f_{0}}{c_{0}}\left(r_{2}-r_{1}\right)\right) \operatorname{si}\left(\pi \frac{B}{c_{0}}\left(r_{2}-r_{1}\right)\right)
\end{aligned}
$$

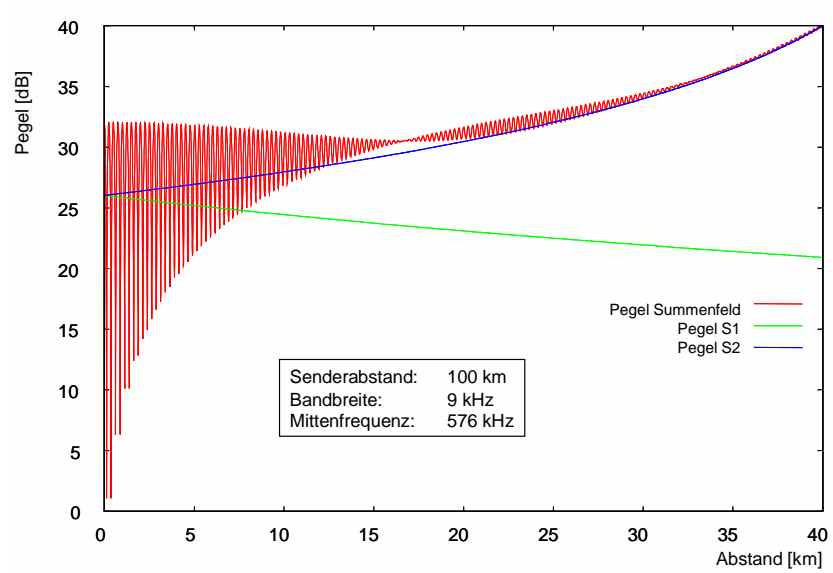

Abb. 3. Beispiel Summenpegel im idealen SFN.

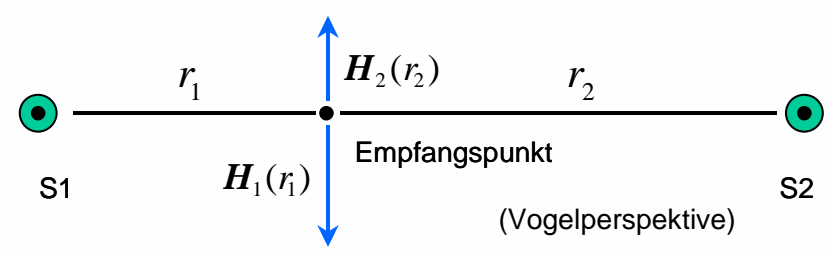

Abb. 4. Überlagerung der H-Felder auf der Verbindungslinie.

In Abb. 3 ist als Beispiel für eine realistische DRMKonfiguration der Pegel des Summenfeldes rot über der Entfernung vom Mittelpunkt der Verbindungsgeraden (Zentrum der Verwirrungszone) zu einem Sender hin aufgetragen. Als einfaches Ausbreitungsmodell wurde Freiraumdämpfung $(\hat{E}(r) \sim 1 / r)$ angenommen. Die Pegel der Einzelsender S1 und S2 sind ebenfalls dargestellt (blau, grün). In der Mitte der Verwirrungszone entstehen positive Überlagerungen mit einem Gewinn von bis zu $6 \mathrm{~dB}$ gegenüber einem Einzelsender aber auch sehr tiefe Einbrüche bis zur fast völligen Auslöschung (Flat-Fading).

Die Breite der Verwirrungszone wird durch die siFunktion in Gleichung (5) bestimmt und ist damit direkt von der Bandbreite $B$ des Signals (1. Nulldurchgang der siFunktion) abhängig. Je größer die Bandbreite, desto kleiner die Verwirrungszone. Die Überlagerungseffekte sind dort am größten, wo die Pegel der Einzelsender etwa gleich groß sind. Der Abstand zweier Einbrüche über der Entfernung vom Mittelpunkt beträgt $\lambda / 2$ und wird direkt durch die Mittenfrequenz $f_{0}$ im cos-Term in Gleichung (5) bestimmt.

Betrachtet man die Überlagerung des Magnetfeldes, so kann man einen ähnlichen Zusammenhang ableiten. In Abb. 4 ist die Überlagerung zweier H-Felder im Gleichwellennetz für den einfachen Fall des Empfangspunktes auf der Verbindungslinie der beiden Sender (von oben gesehen) dargestellt. 


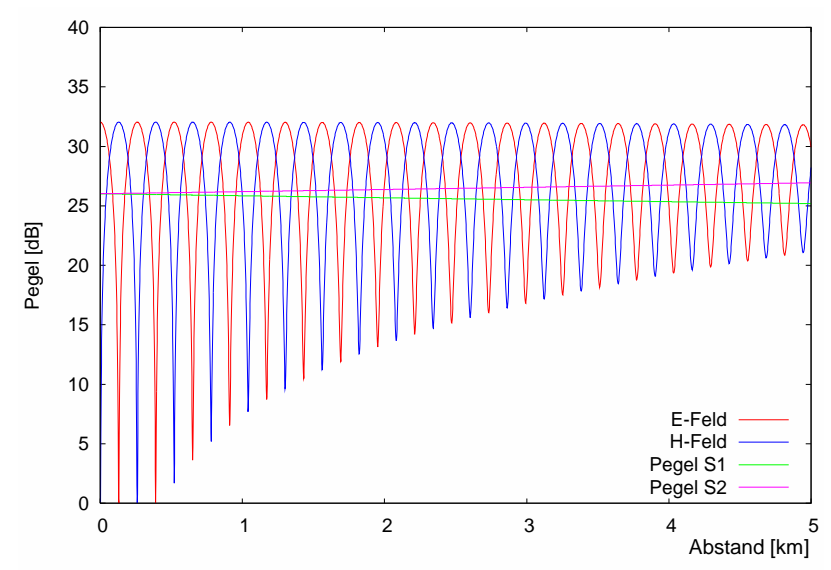

Abb. 5. Konträres Verhalten von E- und H-Feld im SFN.

Im Unterschied zum E-Feld löscht sich das Magnetfeld durch die entgegengesetzte Orientierung der H-Felder im Mittelpunkt zwischen den Sendern gerade aus, wo sich die E-Feld Komponenten optimal addieren. D. h., die E- und HFeld-Komponenten verhalten sich konträr.

Die Gleichung für die verfügbare Leistung des überlagerten H-Feldes (für den einfachen Fall mit einem Empfangspunkt auf der Verbindungslinie zwischen den Sendern) lässt sich damit sofort angeben:

$$
\begin{aligned}
& \frac{P_{12}}{B} \sim \hat{H}_{1}\left(r_{1}\right)^{2}+\hat{H}_{2}\left(r_{2}\right)^{2}+ \\
& -2 \hat{H}_{1}\left(r_{1}\right) \hat{H}_{2}\left(r_{2}\right) \cos \left(2 \pi \frac{f_{0}}{c_{0}}\left(r_{2}-r_{1}\right)\right) \operatorname{si}\left(\pi \frac{B}{c_{0}}\left(r_{2}-r_{1}\right)\right)
\end{aligned}
$$

Ein Ausschnitt des Summenpegels der Überlagerung beider Felder in der Nähe des Mittelpunktes ist in Abb. 5 dargestellt (Parameter wie in Abb. 3). Im Gegensatz zu einem Einzelsender, bei dem die Energien der E- und H-FeldKomponenten im Fernfeld immer gleich groß sind, wird im überlagerten Gleichwellennetz diese Fernfeld-Beziehung aufgehoben (man bewegt sich in einer stehenden Welle). Das H-Feld (blau) hat dort ein Minimum, wo sich das E-Feld (rot) maximal addiert. Dieses Verhalten lässt vermuten, dass eine optimierte Kombinations-Antenne, die gleichzeitig das Eund H-Feld auswertet, im schmalbandigen Gleichwellennetz einen besseren Empfang ermöglicht.

Bewegt man sich von der Verbindungslinie zwischen den beiden Sendern weg, so kann sich das H-Feld wegen der Geometrie nicht mehr komplett auslöschen. Angenommen man benutzte eine omnidirektionale Magnetantenne, so würde der Auslöschungseffekt z.B. auf der Mittelsenkrechten der Verbindungslinie mit dem Abstand zum Mittelpunkt stetig abnehmen. Eine allgemeine Formel für der Überlagerung an beliebigem Empfangspunkt mit omnidirektionaler Magnetantenne lässt sich ebenfalls herleiten.

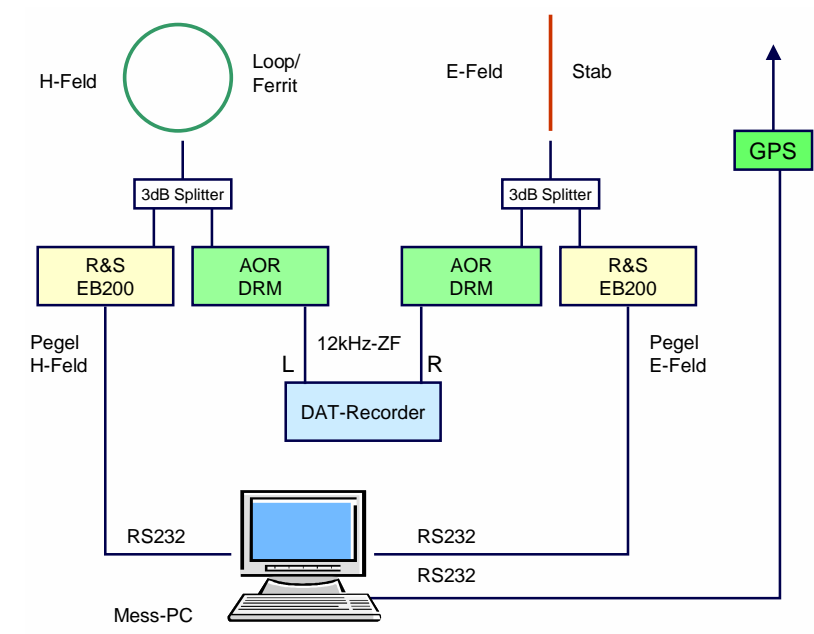

Abb. 6. Messaufbau.

\section{Feldmessungen}

Durch einen DRM-Gleichwellenversuch beim SWR sollten die theoretisch zu erwartenden Überlagerungseffekte verifiziert werden. Im einzelnen wurden folgende Punkte untersucht:

- Pegeleinbrüche innerhalb der Verwirrungszone im ausgeglichenen SFN

- Konträres Verhalten der Überlagerung zwischen E- und H-Feld

- Auswirkungen eines Frequenzversatzes bei stationärem Empfang

- Auswirkung einer Kombi-Antenne (E- und H-Feld)

- Auswirkung der künstlichen Signal-Laufzeit auf die Überlagerungseffekte

\subsection{Messaufbau}

Zum Aufzeichnen der Pegeleinbrüche in der Verwirrungszone und zur Erfassung des konträren Verhaltens von Eund H-Feld wurde ein Messaufbau nach Abb. 6 in einem Messfahrzeug realisiert. Das System bestand aus zwei getrennten Empfangseinrichtungen zur simultanen Pegelmessung und DRM-Auswertung mit jeweis einer magnetischen und einer elektrischen Antenne (Loop/Ferrit, bzw. aktive Stabantenne Fa. R\&S). Zum Einsatz kamen jeweils ein Messempfänger EB200 der Fa. R\&S (selektive Pegelmessung) und ein DRM-Empfänger von AOR mit einem $12 \mathrm{kHz}-$ ZF-Ausgang zur Dekodierung mit einem Software-Radio. Der DRM-ZF-Ausgang des AOR-Empfängers wurde bei den Versuchen mit einem DAT-Recorder aufgezeichnet. Damit konnten die Versuche und Effekte „offline“ am Schreibtisch 


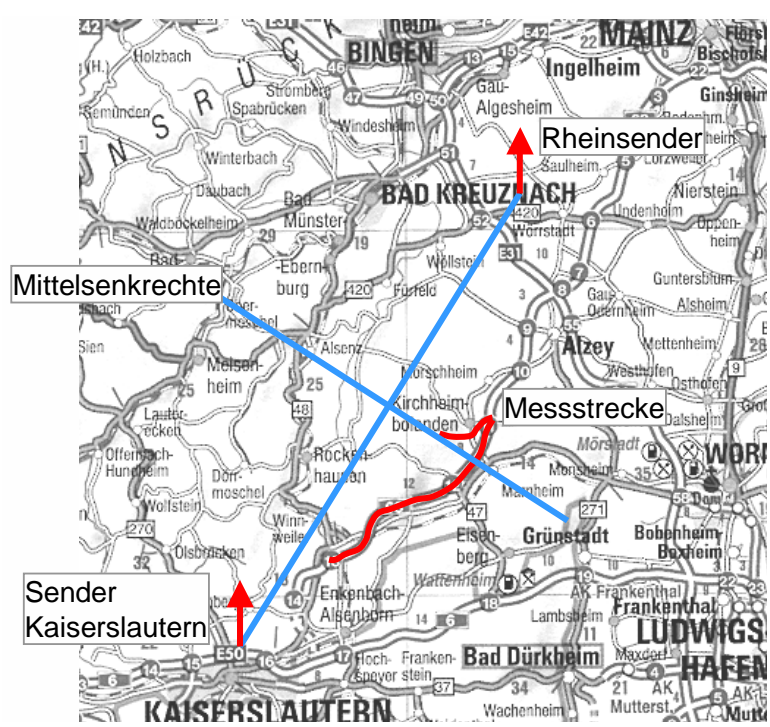

Abb. 7. DRM-Gleichwellennetz mit Messstrecke (rot), Verbindungslinie und Mittelsenkrechter (blau).

nachvollzogen und ausgewertet werden. Die simultane Pegelaufzeichnung wurde mit einem Messprogramm über serielle Schnittstellen mit einem Mess-PC durchgeführt. Zusätzlich konnten auch die GPS-Koordinaten mit aufgezeichnet werden.

\subsection{Das DRM-Gleichwellennetz}

In Abb. 7 ist die räumliche Anordnung des DRMGleichwellennetzes mit zwei vertikal strahlenden Mittelwellensendern auf $1485 \mathrm{kHz}$ zwischen Mainz und Kaiserslautern dargestellt. Neben den Senderstandorten (Rheinsender und Kaiserslautern) sind zusätzlich die Messstrecke (rot) sowie die Verbindungslinie zwischen den Sendern und die zugehörige Mittelsenkrechte (blau) eingezeichnet.

Zunächst musste ein ausgewogenes Gleichwellennetz eingestellt werden. Dazu wurde am Rheinsender die Leistung soweit abgesenkt, dass an einem Referenzpunkt auf der geografischen Mittelsenkrechten zwischen den Sendern gleiche Pegelverhältnisse der Einzelsender vorhanden waren (ca. $1 \mathrm{~dB}$ Unterschied). Als Messstrecke wurde die Autobahn A63 zwischen AS Kirchheim-Bolanden, AS Göllheim und AS Wolfsweiler gewählt. Die 7,5 km lange Strecke zwischen AS Kirchheim-Bolanden und AS Göllheim lag somit im Maximum der zu erwartenden Verwirrungszone.

\subsection{Messergebnisse}

Pegeleinbrüche im E-Feld:

In Abb. 8 sind der Vergleich der zu erwartenden Pegeleinbrüche (E-Feld) und die Ergebnisse der Messfahrt über

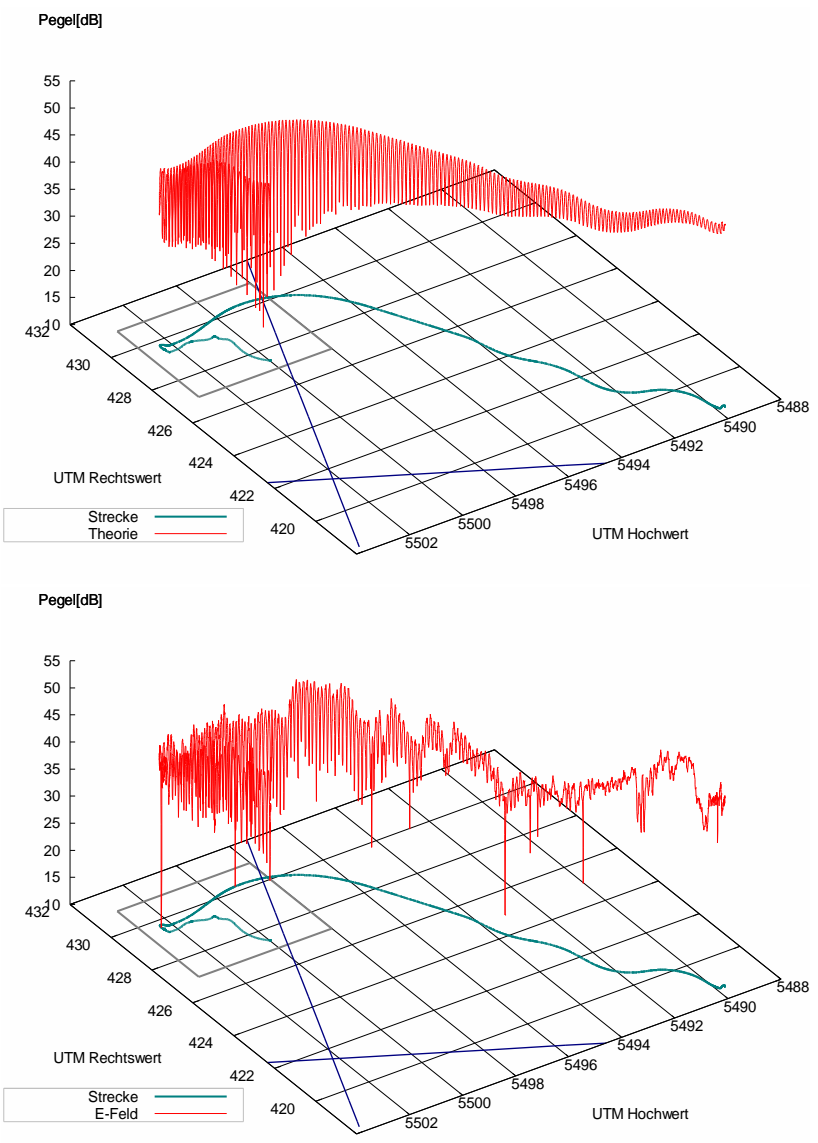

Abb. 8. Vergleich theoretischer und gemessener Pegel.

der Wegstrecke dargestellt. Bei der theoretischen Berechnung wurde als Wellenausbreitungsmodell wieder die Freiraumdämpfung angenommen.

Neben den erwarteten Pegeleinbrüchen durch die Überlagerung in der Verwirrungszone rühren die Extremwerte von Durchfahrten unter Betonbrücken her. Generell ist zu beobachten, dass der Pegel im Mittelwert gegenüber der Prognose deutlich schwankt. Dies liegt an den Ausbreitungsbzw. Empfangsbedingungen der Mittelwellensender (Topographie, Bodenleitfähigkeit). Diese Schwankungen zeigten sich auch bei Pegelmessungen der Einzelsender.

In Abb. 9 ist noch einmal die Theorie und die Messung des überlagerten E-Feldes vergrößert dargestellt (Ausschnittsbereich graues Quadrat). Hier kann man sehr gut die Übereinstimmung zwischen Theorie und Messung erkennen. Man sieht deutlich die prognostizierten Pegeleinbrüche von z.T. über $20 \mathrm{~dB}$.

Konträres Verhalten von E- und H-Feld:

In Abb. 10 sind die Pegel des E- und H-Feldes zusammen über einem Teil der Messstrecke dargestellt. Dabei wurde als x-Achse die Abstands-Differenz zu den Sendern 

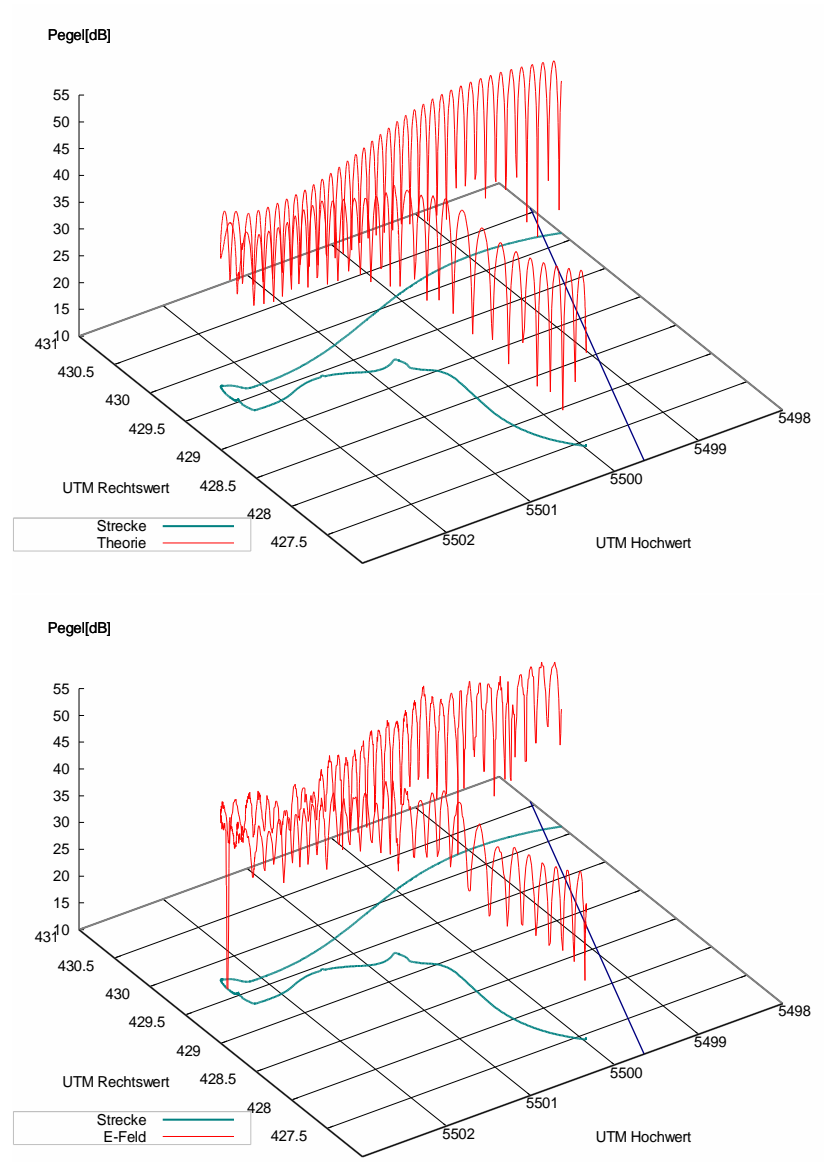

Abb. 9. Vergleich Pegelverlauf im Ausschnitt.

$\left(r_{2}-r_{1}\right)$ berechnet. Der Ausschnitt zeigt dementsprechend eine Strecke von der Mittelsenkrechten $(x=0)$ in Richtung Kaiserslautern.

Das gegenläufige Verhalten zwischen E- und H-Feld ist deutlich zu erkennen. Durch die Richtwirkung der magnetischen Antenne fallen die Einbrüche beim H-Feld etwas geringer aus. Die synchronen Schwankungen des Mittelwertes sind für E- und H-Feld gleich.

\section{Frequenzversatz:}

Bei einem weiteren Test wurde die Frequenz des Rheinsenders gegenüber dem Sender Kaiserslautern um $1 \mathrm{~Hz}$ (kleinste Einstellmöglichkeit) verstellt. (Ab einem Frequenzversatz von $3 \mathrm{~Hz}$ wird durch Orthogonalitätsverletzungen und resultierender Verringerung des Signal-Rauschabstands die Audio-Dekodierung nicht mehr möglich.) An unserem Referenzpunkt auf der Mittelsenkrechten wurde stationär das Eund H-Feld synchron aufgezeichnet. In der Abb. 11 ist das E- und H-Feld über der Zeit aufgetragen.

Im Vergleich von E- und H-Feld erkennt man wieder deutlich das konträre Verhalten der beiden Felder. Die Einbrüche erscheinen im Rhythmus der Offset-Frequenz von

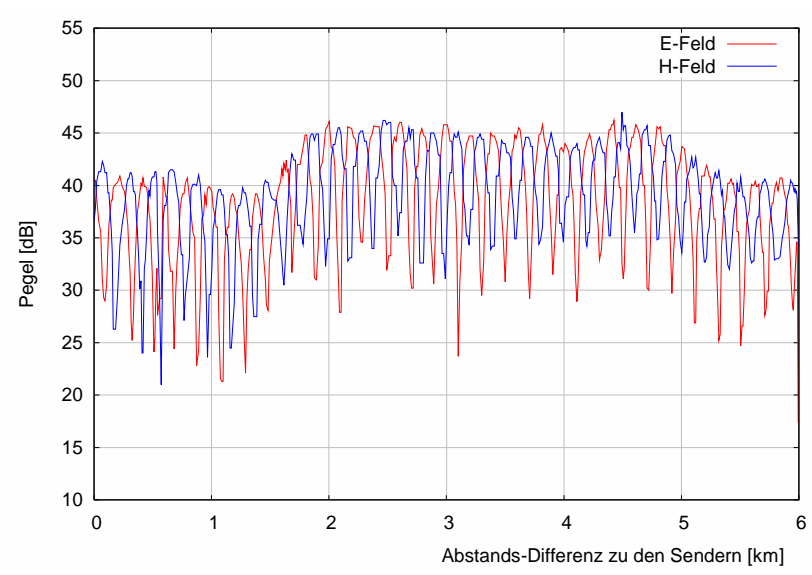

Abb. 10. Pegelverlauf über Teilstrecke für E- und H-Feld.

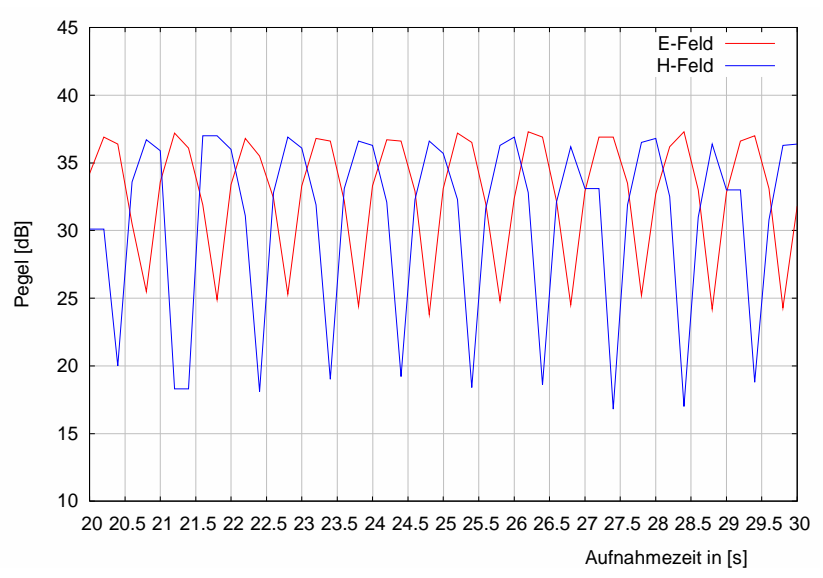

Abb. 11. Pegel bei Frequenzversatz am Referenzpunkt.

$1 \mathrm{~Hz}$. Durch die begrenzte Abtastzeit von $200 \mathrm{~ms}$ sind die Abtastwerte etwa immer an der gleichen Stelle. (Dadurch werden die Extremwerte beim E-Feld nicht aufgelöst. Die maximale Abtastfrequenz ist durch das Messgerät und das Steuerprogramm beschränkt.)

\section{Frequenzversatz und Kombiantenne:}

Bei diesem Test wurde versucht, eine E- und H-Antenne zu kombinieren. Laut Theorie ergibt sich dabei (bei vergleichbaren Signalspannungen der Antennen) eine starke Richtwirkung (siehe z.B. Rothammel, 1989). Durch dieses Richtverhalten der Antenne kann ein Gleichwellensender "ausgeblendet" werden. In der Abb. 12 ist der Pegel der optimierten Kombi-Antenne verglichen mit einer einzelnen magnetischen Antenne dargestellt. Es war beim Abstimmen der Kombi-Antenne durch das Zuschalten von Dämpfungsgliedern fast gelungen die Einbrüche zu eliminieren (Restwelligkeit von $<4 \mathrm{~dB}$ ). Im Vergleich dazu zeigte eine einzelne Magnetantenne Einbrüche von über $20 \mathrm{~dB}$. 


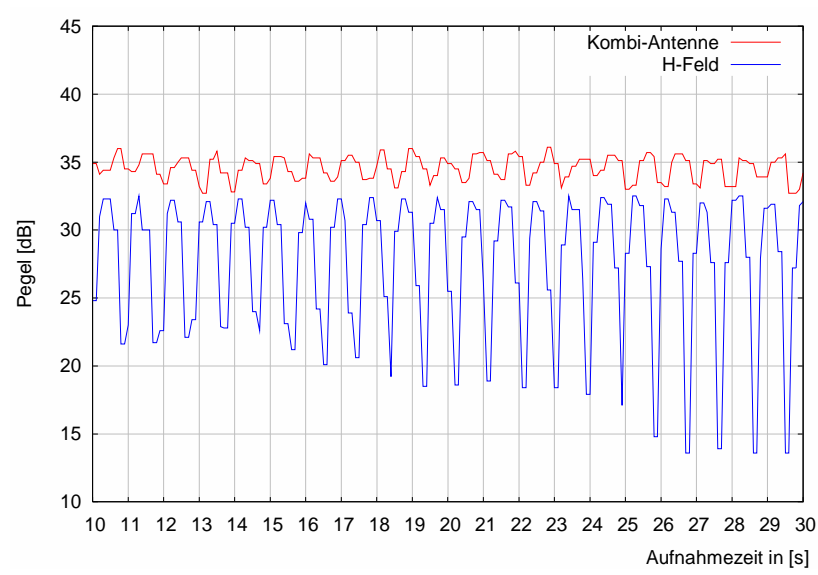

Abb. 12. Kombi-Antenne bei Frequenzversatz.
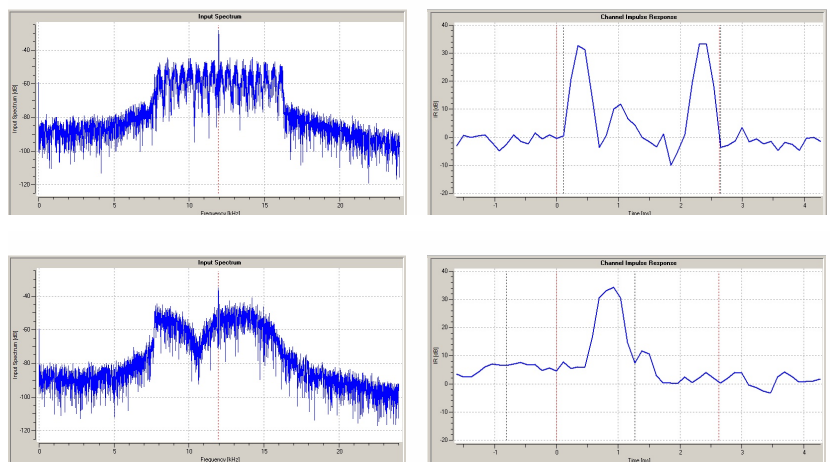

Abb. 13. Spektrum (links) und Impulsantwort (rechts) bei künstlicher Signal-Laufzeit $2 \mathrm{~ms}$ (oben) und 0,2 ms (unten).

Eine ideale Kombi-Antenne sollte die maximale Leistung aus jeweils dem E- oder H-Feld entnehmen und dabei keine Richtwirkung zeigen. Solche Antennen kann man sich als spezielle Diversity-Systeme vorstellen, eine Realisierung in Konsumer-Geräten wäre aber aufwendig und teuer. Diese aufwendige Antenne ist auch nicht notwendig, wenn das Gleichwellennetz richtig eingestellt ist (siehe künstliche Signal-Laufzeit).

Der Frequenzversatz zur Verbesserung der Empfangssituation ist bei einem DRM-Gleichwellennetz nicht zu empfehlen. Die Einbrüche beim stationären Empfang werden zwar zeitlich begrenzt, durch den Frequenzversatz erhält man aber eine Verschlechterung des Signal-Rausch-Abstands durch Orthogonalitätsverletzungen.

\section{Künstliche Signal-Laufzeit:}

Die künstliche Signal-Laufzeit bei DRM-Sendern wird i.d.R. dazu verwendet, um Laufzeitunterschiede bei unterschiedlichen Sendertypen (Herstellern) oder Laufzeiten über

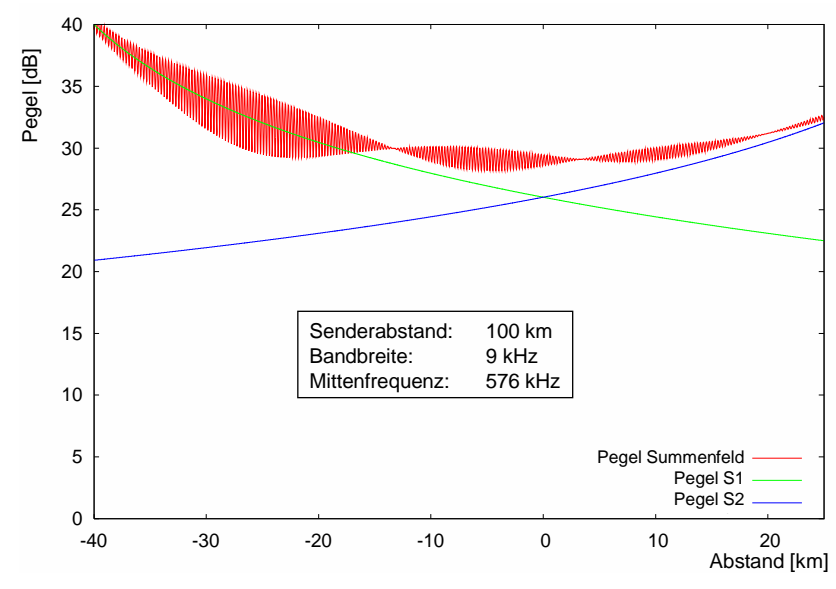

Abb. 14. Pegel E-Feld bei künstlicher Laufzeit 0,2 ms.

unterschiedliche Signalzuführungen zu kompensieren und damit das Gleichwellennetz zu synchronisieren.

Die Aussendung der OFDM-Symbole an den Senderstandorten wird dabei zeitlich verzögert. Damit ändern sich an einem Empfangspunkt auch die Phasenlagen der Einzelträger des DRM-Signals bzgl. eines nicht verzögerten Signals. Dadurch hat die künstliche Laufzeitverschiebung einen direkten Einfluss auf die Überlagerung der DRMSignale in der Verwirrungszone.

Bei einem Test im Verwirrungsgebiet wurde bei etwa gleichen Pegelverhältnissen die Laufzeit des Rheinsenders stufig von $2 \mathrm{~ms}$ bis $0,2 \mathrm{~ms}$ verändert und das Spektrum beobachtet. Das Ergebnis ist in Abb. 13 zu sehen.

Im Spektrum lassen sich jeweils deutlich die selektiven Pegeleinbrüche erkennen. Der Abstand der Einbrüche im Spektrum ist umgekehrt proportional zur Laufzeitdifferenz $\Delta t$ der beiden Signalanteile. Trotz der starken selektiven Pegeleinbrüche, war im Versuch der Gesamtpegel des Nutzsignals stets ausreichend und dadurch, dank der vorhandenen Schutzmechanismen bei DRM (Frequenzund Zeitinterleaving), eine störungsfreie Audio-Dekodierung möglich. Erst wenn die Bedingung zur Kohärenzbandbreite nicht mehr erfüllt ist $(1 / \Delta t \ll B$, z.B. bei $B=9 \mathrm{kHz}$ und $\Delta t<0,01 \mathrm{~ms}$ ) entsteht das befürchtete Flat-Fading mit totalen Auslöschungseffekten.

Theoretischer Einfluss der Signal-Laufzeit:

Durch den gezielten Einsatz der Signal-Laufzeit lässt sich die Verwirrungszone räumlich "verschieben". Dabei kann man das Gleichwellennetz so einstellen, dass die Zone mit etwa gleichen Phasenlagen der Einzelträger (Laufzeitdifferenz $\Delta t=0$ ) in einem Bereich zu liegen kommt, in dem die Pegelunterschiede so groß sind, dass extreme Pegeleinbrüche nicht mehr auftreten. Brücksichtigt man den Parameter der künstlichen Laufzeit $d t$ jedes Senders in der Berechnung zur bandbreitenbezogenen verfügbaren Gesamtleistung 
(Gleichung 5) so erhält man (für das E-Feld) folgende Formel zur Abschätzung des Gesamtpegels:

$$
\begin{aligned}
\frac{P_{12} \sim}{B} \sim & \hat{E}_{1}\left(r_{1}\right)^{2}+\hat{E}_{2}\left(r_{2}\right)^{2}+2 \hat{E}_{1}\left(r_{1}\right) \hat{E}_{2}\left(r_{2}\right) . \\
& \cdot \cos \left(2 \pi \frac{f_{0}}{c_{0}}\left(\left(r_{2}+c_{0} d t_{2}\right)-\left(r_{1}+c_{0} d t_{1}\right)\right)\right) . \\
& \cdot \operatorname{si}\left(\pi \frac{B}{c_{0}}\left(\left(r_{2}+c_{0} d t_{2}\right)-\left(r_{1}+c_{0} d t_{1}\right)\right)\right)
\end{aligned}
$$

In Abb. 14 ist der zu erwartende Pegelverlauf des E-Feldes bei einer künstlichen Signal-Laufzeit des Senders S1 von $0,2 \mathrm{~ms}$ bei sonst gleichen Parametern wie in Abb. 3 dargestellt.

Man sieht deutlich die Verschiebung und Abflachung der Pegeleinbrüche (Maximum der si-Funktion) vom Mittelpunkt weg. Bei größeren künstlichen Signal-Laufzeiten werden die Einbrüche noch geringer. Somit ist eine gezielte Einstellung der Signal-Laufzeiten die einfachste Methode, um das befürchteten Flat-Fading in der Verwirrungszone zu vermeiden.

\section{Schlussfolgerung}

Die gemessenen Überlagerungseffekte im realen Gleichwellennetz entsprachen den theoretischen Erwartungen. Zur Bewältigung der Überlagerungsprobleme wurden verschiedene Lösungsansätze untersucht.
In den Feldmessungen hat sich gezeigt, dass ein FrequenzOffset keine merklichen Empfangsverbesserungen erzielt. Mit einer Kombiantenne (Ausnutzung von E- und H-Feld) konnte der nutzbare Empfangspegel erhöht werden, eine Umsetzung erscheint aber aufwendig und teuer.

Durch das gezielte Einstellen der Signallaufzeiten an den Sendern kann das befürchtete Flat-Fading im Verwirrungsgebiet vermieden werden. Mit der künstlichen Signallaufzeit steht dem Netzbetreiber ein wichtiger Planungsparameter zur verfügung, um einen robusten Gleichwellenbetrieb zu realisieren. Die Gleichwellentechnik für Schmalband-DRM ist dadurch praktikabel und sinnvoll.

\section{Literatur}

ETSI Standard: ETSI ES 201980 Digital Radio Mondiale (DRM) System Specification, http://www.etsi.org, 2004-2006.

Meinke-Gundlach: Taschenbuch der Hochfrequenztechnik Band 1 (Hrsg. Lange, K. und Löcherer, K.-H.), Springer-Verlag, Berlin/Heidelberg, B4, 1992.

Rothammel, K.: Antennenbuch 11. Auflage, Frankh, Stuttgart, 1989.

Mäusel, R. und Göbel, J.: Analoge und digitale Modulationsverfahren, Hüthig, Heidelberg, S. 233ff, 2002. 